\title{
Modeling and Dynamics of Nonlinear Acoustic Waves in a Combustion Chamber
}

\author{
N. Ananthkrishnan ${ }^{1}$ and Shardul Deo ${ }^{2}$ \\ Indian Institute of Technology (Bombay), Mumbai 400076, India \\ and \\ Fred E.C. Culick ${ }^{3}$ \\ California Institute of Technology, Pasadena, CA 91125
}

\begin{abstract}
Future combustors designed for better efficiency and lower pollutant emission are expected to operate closer to their stability boundary, thereby increasing the risk of encountering combustion instability. Onset of combustion instability leads to limit cycle oscillations in the acoustical fluctuations that can often reach amplitudes large enough to cause severe damage. Active control strategies are, therefore, being considered to prevent combustion instabilities, but their development requires nonlinear models that can faithfully capture the combustor system dynamics. A framework for the approximate analysis of the nonlinear acoustics in a combustion chamber exists, which includes all relevant linear contributions and also second order gasdynamic nonlinearities. Nonlinear combustion effects in the form of pressure and velocity coupling models have also been incorporated into the analysis with the aim of capturing the phenomenon of triggered instability, where the acoustical fluctuations are linearly stable to small perturbations, but show a limit cycle behavior for larger perturbations. However, several questions such as those relating to 1) modal truncation of the equations for the acoustic dynamics, 2) absence of triggered limit cycles in the formulation with only second order gasdynamic nonlinearities, and 3) the form of the velocity coupling function, including the need for a threshold character, have not been satisfactorily resolved. In this paper, we address some of these questions on modeling and dynamics of acoustic waves in combustion chambers, using the approximate analysis, that have remained unanswered over the years.
\end{abstract}

\section{Introduction}

Combustion chambers operating under conditions that favor high efficiency and low pollutant emission are prone to suffer from combustion instabilities. These instabilities arise due to a coupling between the unsteady fluid motion and the combustion processes in the chamber. This interaction can be thought of as analogous to a positive feedback mechanism where fluctuations in the flow properties and in the combustion heat release drive each other in a regenerative manner. Amplitude dependent nonlinearities then cut in to limit the growth in the fluctuations. Thus, to an observer external to the chamber, combustion instabilities appear as self-excited limit cycle oscillations in the flow variables. It was shown by Chu and Kovasznay [1] that the fluctuations could be represented by a combination of acoustic, vortical, and entropic waves propagating in the combustion

\footnotetext{
${ }^{1}$ Assistant Professor, Department of Aerospace Engineering, Senior Member AIAA, akn@aero.iitb.ac.in

${ }^{2}$ Undergraduate Student, Department of Aerospace Engineering, deo@aero.iitb.ac.in

${ }^{3}$ Richard L. and Dorothy M. Hayman Professor of Mechanical Engineering and Professor of Jet Propulsion, Fellow AIAA, fecfly@caltech.edu
} 
chamber. Traditionally, the term combustion instability has been used to refer to instabilities in the acoustical fluctuations. These acoustic oscillations can often reach amplitudes large enough to cause severe damage, and sometimes even complete failure. As a result, considerable effort has been made in the past to predict the onset of acoustic instabilities in combustion chambers. Solid propellant rocket motors have been known to exhibit two qualitatively different kinds of behavior at onset of combustion instability: 1) linear instability, where the acoustical fluctuations, in response to a small perturbation, build up to a limit cycle, and 2) nonlinear instability, where the acoustical fluctuations show a stable, damped response for small perturbations, but show a limit cycle response to larger perturbations. Nonlinear instability of this nature has been called pulsed or triggered instability $[2,3]$. Both linear and nonlinear (triggered) instability have been observed in recent experiments on a gas turbine combustor as well [4].

Much of the early work on combustion instability dealt with the question of linear stability, assuming that the acoustic oscillations could be described in terms of the classical acoustic modes of the combustion chamber. The linear combustion instability problem appears to have been satisfactorily addressed, though work continues on the effects of the vorticity and entropy waves in the combustion chamber on the stability of the acoustic oscillations [5, 6]. It was, however, recognized early on that the dynamics of acoustic waves in combustion chambers was essentially nonlinear, and that it was necessary to be able to understand and model the nonlinear behavior of these acoustic waves. The subject of nonlinear acoustics in combustion chambers began to be addressed by several researchers in the 1960's and 1970's. Most of these developments have been reviewed by Culick $[7,8]$, and are, therefore, not repeated here. The work by Culick $[9,10]$ during this period provided a general framework for the analysis of the nonlinear dynamics and stability of acoustic waves in combustion chambers. Under this framework, the partial differential equations of fluid conservation were approximated by a spatial averaging procedure to a set of ordinary differential equations for the amplitudes of the acoustic modes. The approximations in the analysis, and the derivation of the equations for the acoustic modes, have been described on several occasions $[7,8]$. The equations for the modal amplitudes form a set of coupled, nonlinear differential equations that account for all relevant linear processes and include contributions from nonlinear gasdynamics to second order. On truncating the equations to a finite number of modes, the modal equations could be solved for the time evolution of the acoustic oscillations.

Culick and Yang [11] reported a numerically computed solution for the acoustic oscillations with a five-mode truncation of the approximate equations that was found to compare reasonably with a more exact numerical solution [11]. Conditions for the existence and stability of the limit cycle oscillations, and the qualitative dependence of the limit cycles on the system parameters, for the case of two longitudinal acoustic modes, were reported by Awad and Culick [12], and by Paparizos and Culick [13]. However, none of these studies were able to demonstrate triggered instability. It was felt, but not conclusively established, that the approximate analysis with nonlinear, second order gasdynamics alone was not capable of showing triggered instability. Extensions of the formulation to include third order gasdynamic nonlinearities and higher order interactions between the mean flow and the acoustics were equally unsuccessful in capturing triggered instability [14] in contrast to earlier results reported by Zinn [15]. The difference between the two conclusions remains unexplained. Nonlinear combustion models then remained the most attractive candidate to represent triggered instability within the framework of the approximate analysis. Unsteady combustion 
in solid propellant rockets had already been described in terms of pressure and velocity coupling models [16]. Numerical studies by Levine and Baum [17] with an ad hoc velocity coupling model showed that triggered instabilities could indeed be found when nonlinear combustion processes were accounted for. However, the form of the velocity coupling function was purely empirical and it was difficult to judge its validity or uniqueness. Also, it was not easy to arrive at qualitative conclusions regarding the conditions for onset of triggered instability from numerical simulations alone.

A significant step forward in the investigations came with the introduction of the methods of modern dynamical systems theory by Jahnke and Culick [18] to the analysis of nonlinear combustion instabilities. By using a continuation algorithm, it became possible to systematically and efficiently compute all steady state and limit cycle solutions over a range of parameter values. Stability of each steady state and limit cycle solution could be numerically established, and points of onset of instability could be identified with bifurcations. The qualitative behavior of the acoustic waves at onset of combustion instability then depends on the type of bifurcation, and on the nature of the limit cycles that emerge at the bifurcation point. This information is usually represented in the form of a plot of steady state values (peak amplitude in case of a limit cycle) against a suitable parameter in a bifurcation diagram. For longitudinal acoustic modes in a combustion chamber of uniform cross section, Jahnke and Culick [18] showed that results from a two-mode approximation were qualitatively dissimilar to those from a four- or six-mode approximation. However, they could draw no conclusion about the number of acoustic modes that need to be retained in their analysis, nor did their computations with second order gasdynamics alone display triggered limit cycles. Culick et al [19] extended the work in [18] by including the nonlinear combustion model of Levine and Baum [17] in addition to the second order gasdynamic nonlinearities. They confirmed that the nonlinear velocity coupling term in the Levine-Baum model did induce triggered limit cycles. However, their results with and without time-averaging showed significant discrepancies. Their studies also suggested that a four-mode approximation could satisfactorily capture the qualitative dynamics for the case of a first mode instability. Experiments by Ma et al [20] have suggested that the velocity coupling function has a threshold nature, i.e., there is a threshold value of the acoustic velocity below which the effects of nonlinear combustion are not felt. In a recent paper, using a four-mode truncation and an ad hoc threshold velocity coupling model, Burnley and Culick [21] have computed a bifurcation diagram which shows triggered limit cycles. Additional results are available in the thesis by Burnley [22].

Despite the impressive progress over the last three decades in modeling the nonlinear dynamics of acoustic waves in combustion chambers, many questions yet remain unanswered. For example, first of all, there is still no convincing argument for how many modes need to be retained in a truncated model of the coupled oscillator equations in order to predict the qualitative dynamics of the acoustic modes correctly. Secondly, though widely believed, it has never been definitely established that the approximate analysis with second order gasdynamics alone could not show triggered instability. Thirdly, the form of the velocity coupling function and, in particular, the need for a threshold character, has not been satisfactorily explained. These and such other questions have gained significance in the light of recent focus on active control of combustion instabilities [23, 24]. Rather than restrict operation to safe, stable regions at the cost of decreased performance, future combustors seeking higher performance will be operated closer to their stability boundary, thereby increasing the risk of encountering combustion instability. Active control strategies promise to 
provide a feasible solution to the problem of preventing instability in these combustors, but they are expected to depend heavily on nonlinear models that can successfully capture the qualitative features of the combustion system dynamics. The approximate formulation developed by Culick and his co-workers appears to provide a suitable framework for the development of active control laws for combustion instability, but there is a need to address questions such as those listed above before the Culick framework can be confidently applied to devise active combustion control strategies. Recent developments $[25,26,27]$ in the use of bifurcation theory for the modeling of large-amplitude limit cycle oscillations have made it possible to seek answers to some questions on the qualitative dynamics of the acoustic waves at onset of combustion instability.

In the remainder of this paper, we first closely examine the coupled oscillator equations for the acoustic modal amplitudes. A careful study of the energy transfer between the acoustic modes provides a clue to the number of modes that need to be retained for a qualitatively correct analysis of the limit cycles at onset of combustion instability. The minimum order of the modal truncation for the first and second mode instability cases is determined, thereby resolving a longstanding issue in the modeling of acoustic waves in combustion chambers. Following this, two known mechanisms for triggered instability in coupled oscillator systems are briefly reviewed. With this knowledge, we then explain the lack of triggered limit cycles in the approximate formulation containing only second order gasdynamic nonlinearities. This is a result that, though widely believed in the past, has been theoretically established here for the first time. Finally, nonlinear combustion mechanisms for triggering are studied. Observations by Culick et al [19] that nonlinear pressure coupling does not lead to triggering are now explained. Velocity coupling models used in the past are evaluated and are found to display non-physical dynamical behavior. The need for a threshold velocity coupling function is critically examined and a new form of the velocity coupling function is derived that naturally shows a threshold character. The approach at all times is from the viewpoint of the qualitative theory of dynamical systems. However, numerical results are provided to illustrate the conclusions arrived at from the theory.

\section{Coupled Oscillator Equations}

The nonlinear dynamics of acoustic waves in a combustion chamber has been modeled by Culick [10] as a set of coupled second order oscillators, one for each acoustic mode. For the case of a combustion chamber of uniform cross section as considered in $[18,19]$, the modal natural frequencies can be assumed to be integral multiples of the primary acoustic mode frequency. Then, the coupled oscillator equations, with time non-dimensionalized by the primary mode frequency, can be written as follows:

$$
\begin{aligned}
\ddot{\eta}_{n}-2 \hat{\alpha}_{n} \dot{\eta}_{n}+n\left(n-2 \hat{\theta}_{n}\right) \eta_{n}= & -\sum_{i=1}^{n-1}\left(\hat{C}_{n i}^{(1)} \dot{\eta}_{i} \dot{\eta}_{n-i}+\hat{D}_{n i}^{(1)} \eta_{i} \eta_{n-i}\right) \\
& -\sum_{i=1}^{\infty}\left(\hat{C}_{n i}^{(2)} \dot{\eta}_{i} \dot{\eta}_{n+i}+\hat{D}_{n i}^{(2)} \eta_{i} \eta_{n+i}\right)
\end{aligned}
$$

where $\eta_{n}$ is the amplitude of the $n^{\text {th }}$ acoustic mode. Equation (1) includes linear contributions from combustion processes, gas-particle interactions, boundary conditions, and interactions between the steady and unsteady flow fields. Additionally, Eq. (1) also includes contributions from nonlinear 
gasdynamics to second order as given by the quadratic terms on the right hand side, where the coefficients $\hat{C}, \hat{D}$ are as follows:

$$
\begin{aligned}
& \hat{C}_{n i}^{(1)}=\frac{-1}{2 \gamma i(n-i)}\left[n^{2}+i(n-i)(\gamma-1)\right] \\
& \hat{C}_{n i}^{(2)}=\frac{1}{\gamma i(n+i)}\left[n^{2}-i(n+i)(\gamma-1)\right] \\
& \hat{D}_{n i}^{(1)}=\frac{\gamma-1}{4 \gamma}\left[n^{2}-2 i(n-i)\right] \\
& \hat{D}_{n i}^{(2)}=\frac{\gamma-1}{2 \gamma}\left[n^{2}+2 i(n+i)\right]
\end{aligned}
$$

The parameters $\hat{\alpha}_{n}$ and $\hat{\theta}_{n}$ in Eq. (1) are defined as

$$
\hat{\alpha}_{n}=\alpha_{n} / \omega_{1}, \quad \hat{\theta}_{n}=\theta_{n} / \omega_{1}
$$

where $\omega_{1}$ is the natural frequency of the first acoustic mode. For a cylindrical chamber of $L / D=$ 11.8 , the first mode frequency, $\omega_{1}$, is $5654.86 \mathrm{rad} / \mathrm{s}$, and typical values for the linear growth rates, $\alpha_{n}$, and frequency shifts, $\theta_{n}$, are as given in Table 1 . It can be seen from Table 1 that the modes are generally lightly damped and have only small frequency shifts, which means that the shifted modal frequencies remain appoximately integral multiples of the shifted primary mode frequency. It may also be noticed that the oscillators in Eq. (1) are linearly uncoupled, but are coupled through the nonlinear gasdynamic terms. In particular, it will be seen later that the quadratic terms on the right hand side of Eq. (1) with coefficients $\hat{C}^{(1)}$ and $\hat{D}^{(1)}$ ensure that the set of oscillators is resonantly coupled. For a set of resonantly coupled, lightly damped, nonlinear oscillators as in Eq. (1), it is not immediately obvious as to which modes affect the stability of a particular mode. Therefore, the question of how many higher order modes need to be retained for a correct solution of the $n^{\text {th }}$ mode instability problem is not easy to answer. Previous numerical results [18, 19] suggest that when too few modes are retained in an analysis, the qualitative predictions of the nonlinear dynamic behavior at instability may be incorrect. At the same time, inclusion of higher order modes beyond a point does not seem to have a significant influence on the quantitative accuracy. Both these observations are not surprising, but 'How few (modes) is too few?' is a question that has not received a satisfactory answer to date.

\subsection{Energy Transfer}

Looking at Eq. (1), it is clear that in the absence of the second order gasdynamic terms on the right hand side, the individual modes behave as uncoupled linear oscillators. The coupling, and hence the energy transfer, between the modes is entirely due to the nonlinear gasdynamics. The influence of the nonlinear terms in the energy transfer process is presented in a concise form in Table 2 for the first eight modes. Each entry in the second column of Table 2 represents a pair of nonlinear gasdynamic terms that transfer energy to a particular mode from a lower numbered mode, i.e., energy transfer up the mode numbers from lower to higher modes. The third column, on the other hand, lists the terms that cause reverse energy transfer, i.e., from higher mode numbers to the lower ones. For instance, in the row for mode number 2, a term ' 13 ' implies that the second mode is excited by terms of the form $\eta_{1} \eta_{3}$ and $\dot{\eta_{1}} \dot{\eta_{3}}$. The term ' 13 ' represents a reverse transfer of energy from the third mode to the second mode. It can be seen that terms with $\hat{C}^{(1)}$ and $\hat{D}^{(1)}$ as 
coefficients appear in the second column of Table 2, while the third column consists of terms with $\hat{C}^{(2)}$ and $\hat{D}^{(2)}$ as coefficients.

A look down the second column of Table 2 shows that every term in the energy transfer up the modes acts as a near-resonant excitation. For instance, consider the first mode to be oscillating at its (shifted) natural frequency $\omega_{1}^{s}$, and recall that the (shifted) natural frequencies of the higher modes are approximately integral multiples of that of the first mode. Then, the terms ' 11 ' will excite the second mode at exactly $2 \omega_{1}^{s}$, which is approximately equal to its (shifted) natural frequency. Likewise, terms like ' 12 ' and ' 21 ' will excite the third mode at a frequency $3 \omega_{1}^{s}$, which will nearly resonate with its (shifted) natural frequency, and so on. Hence, the modes in Eq. (1) represent a set of resonantly coupled oscillators. In contrast, the reverse energy transfer terms in the third column of Table 2 typically excite the modes at frequencies much higher than their resonant frequency, and hence contribute little to the overall modal amplitudes, except for the terms in boldface. The boldface terms represent parametric excitations which can alter the dynamics of the modes, in contrast to the other terms which act as external forcings that can merely contribute to the modal amplitude at a particular frequency. When the boldfaced terms are taken to the left hand side of the respective oscillator equation in Eq. (1), they can be seen to alter the frequency and damping of the modes, and thus, they can change the qualitative dynamical behavior. This can be clearly seen, for example, by writing the dynamical equation for the first mode with the parametric excitations moved to the left hand side as follows:

$$
\ddot{\eta}_{1}+\left[-2 \hat{\alpha}_{1}+\hat{C}_{11}^{(2)} \dot{\eta}_{2}\right] \dot{\eta}_{1}+\left[\left(1-2 \hat{\theta}_{1}\right)+\hat{D}_{11}^{(2)} \eta_{2}\right] \eta_{1}=[\text { other RHS terms }]
$$

The term involving $\hat{C}_{11}^{(2)}$ represents a reverse transfer of energy from the second to the first mode that could potentially destabilize the first mode. The effect of the other boldface terms in Table 2 can be similarly interpreted as a change in the damping and frequency of the oscillator equation in which they appear.

\subsection{Modal Truncation}

We are now in a position to answer the question of how many modes need to be retained in an analysis to obtain qualitatively correct results for an $n^{\text {th }}$ mode instability. Consider the case where the first mode goes linearly unstable and begins oscillating at a frequency $\Omega$ (which may be slightly different from its shifted natural frequency $\omega_{1}^{s}$ due to nonlinear effects). Energy is then transferred to the second mode, which is resonantly excited by the terms ' 11 ' and set into oscillation at a frequency of $2 \Omega$. A part of the energy from the second mode is reverse transferred to the first mode due to the boldfaced ' 12 ' terms in Table 2. Thus, the dynamics of the first mode is nonlinearly coupled to that of the second mode, and it is necessary to consider the first and second mode oscillators coupled together. However, since the parametric excitation terms ' 12 ' can significantly alter the dynamics of the first mode, it is important that they are correctly represented. When the third and higher modes are neglected, the second mode cannot transfer energy up the mode numbers as per the terms in the second column of Table 2. Instead, it is forced to reverse transfer part of this energy to the first mode, due to which the parametric excitations are larger than they ought to be, and the resulting dynamics may show large-amplitude limit cycles that are spurious. Such spurious limit cycles were observed, but could not be explained, in the two-mode continuation results of Jahnke and Culick [18], and, previously, in the two-mode analytical results reported by Awad and 
Culick [12], and by Paparizos and Culick [13]. Hence, the modal truncation should be such that all significant energy transfers up the modes are accommodated. This requires that all modes that directly receive energy from modes 1 and 2, individually or collectively, should be represented in the truncated set of equations. It is seen from Table 2 that direct energy transfer from modes 1 and 2 to the higher modes occurs through the terms ' 12 ' and ' 21 ' to the third mode, and through the term ' 22 ' to the fourth mode. All other energy transfers to the higher modes are indirect in the sense that they require the participation of the first/second mode and another higher mode. Thus, in a first mode instability, the third and fourth modes play an important role as energy sinks and must be included in the modal truncation, even though their direct influence on the dynamics of the first mode is not significant. In summary, the truncated set of equations for correct qualitative analysis of a first mode instability should contain at least four modes - the unstable mode (mode 1), the coupled modes (mode 2), and the energy sinks (modes 3 and 4). This argument can be easily extended to determine the minimum order of the modal truncation for analysis of an $n^{\text {th }}$ mode instability. For example, in case of a second mode instability, it can be shown that the modal truncation must retain at least the first eight modes.

Continuation results for first and second mode instability have been reported in [18, 19, 22], where upto sixteen modes have been retained. Examination of these results confirms that computations for first mode instability with a modal truncation that did not retain at least four modes were qualitatively incorrect. Similarly, second mode instability computations that retained fewer than eight modes are seen to be qualitatively inconsistent. In the following sections of this paper, we shall examine the first mode instability problem with a four-mode truncation. In particular, we shall be interested in conditions under which triggered limit cycles occur.

\section{Triggered Limit Cycles}

The set of coupled acoustic oscillators in Eq. (1) can be seen to have an equilibrium state where each $\eta_{n}=0$, i.e., none of the acoustic modes are excited. Since the oscillators are all linearly decoupled, the linear damping coefficient for each mode, $\hat{\alpha}_{n}$, determines whether or not that particular mode is linearly stable. When each $\hat{\alpha}_{n}$ is negative, then the equilibrium state of the set of acoustic oscillators is linearly stable. In that case, small perturbations from the equilibrium state damp out with time, and the system of oscillators tends to return to its equilibrium state. Combustion instability occurs when one of the modes gets undamped, i.e., the corresponding $\hat{\alpha}_{n}$ changes sign from negative to positive. For positive $\hat{\alpha}_{n}$, the equilibrium state is linearly unstable, and small perturbations in the acoustic mode amplitudes initially grow with time. Nonlinear effects then become important and the modal amplitudes eventually settle down to a periodic oscillation called a limit cycle. Thus, given a model for the nonlinear dynamics of the acoustic waves, such as that in Eq. (1), one needs to predict the amplitude and frequency of the limit cycle oscillations at onset of combustion instability. This is easily done by using a continuation and bifurcation software such as AUTO97 [28]. Consider the first mode instability problem with a four-mode truncation of the set of oscillators in Eq. (1), where the only nonlinear terms are due to second order gasdynamics. Equilibrium states and limit cycles for this case have been computed for varying values of first mode

damping parameter $\hat{\alpha}_{1}$ using the data in Table 1. Results are obtained for the amplitudes of the first four modes and, in case of limit cycles, also the time period of the oscillation. Of these, the first mode amplitude is plotted in Fig. 1 (plots for the other modal amplitudes are qualitatively similar) 
over a range of values of the parameter $\hat{\alpha}_{1}$. Figure 1 shows that the zero-amplitude equilibrium is linearly stable for $\hat{\alpha}_{1}<0$ and becomes unstable for $\hat{\alpha}_{1}>0$, with onset of instability occuring at $\hat{\alpha}_{1}=0$. Stable limit cycles emerge at the critical point $\hat{\alpha}_{1}=0$, which is called a supercritical Hopf bifurcation point. For any negative value of $\hat{\alpha}_{1}$, the only stable solution is the zero-amplitude equilibrium, and the acoustic waves tend to damp out, no matter how large the initial perturbation. Thus, the coupled oscillator model with second order gasdynamics alone shows only linear instability.

Solid propellant rockets, as discussed earlier, have been known to show both linear and nonlinear or triggered instability. Examples of triggered instability are shown in the schematic bifurcation diagrams in Fig. 2, where $x$ is a variable and $\mu$ is a parameter. In each of the diagrams in Fig. 2, there is a range of values of the parameter $\mu$ for which a stable equilibrium state co-exists with a stable limit cycle. For any parameter value in this range, small perturbations in $x$ from the equilibrium state will tend to decay with time, but for larger perturbations, the system may show stable limit cycle oscillations. That is, though the equilibrium state is linearly stable, the system could be pulsed or triggered into limit cycle behavior. These stable limit cycles are called triggered limit cycles. The triggered limit cycles in Fig. 2 are qualitatively different from the stable limit cycles in Fig. 1 in two respects: 1) Triggered limit cycles in Fig. 2 exist to the left of the Hopf bifurcation point that signifies onset of (linear) combustion instability, whereas the stable limit cycles in Fig. 1 occur only for parameter values to the right of the Hopf bifurcation. 2) Moving along the parameter axis from left to right, the triggered limit cycles in Fig. 2 begin abruptly with a finite non-zero amplitude at a fold bifurcation, as against the stable limit cycles in Fig. 1 whose amplitude starts from zero at the Hopf bifurcation point. Triggered limit cycles are, therefore, also called large-amplitude limit cycles in the literature $[25,26,27]$. The onset of triggered limit cycles at a fold bifurcation can be considered to be a nonlinear combustion instability phenomenon. Thus, with increasing values of the parameter $\mu$, the systems in Fig. 2 first show a nonlinear combustion instability at a fold bifurcation, and then a linear combustion instability at a Hopf bifurcation.

Triggered limit cycles can be a disquieting phenomenon due to the sudden increase in the amplitudes of the acoustic modes. Moreover, the phenomenon is worrisome because triggered instability could occur even when linearly stable operating conditions have been ensured. Hence, there is a need to develop models that can faithfully capture the qualitative dynamics of triggered limit cycles in combustion chambers. Unfortunately, the coupled acoustic oscillator model in Eq. (1), from past experience and as seen in Fig. 1, does not seem to accommodate triggered limit cycles, but this has never been definitely established. We can now explain the lack of triggered limit cycles in the oscillator model of Eq. (1) by comparing the influence of the second order gasdynamic nonlinearities with nonlinear terms that are known to cause triggering in coupled oscillator systems. There are two known mechanisms for generation of triggered limit cycles in systems of resonantly coupled oscillators.

1. Nonlinear damping terms of the form $\left|f\left(\eta_{n}\right)\right| \dot{\eta}_{n}$ or $\left|f\left(\dot{\eta}_{n}\right)\right| \dot{\eta}_{n}$ have been shown to produce triggered limit cycles with either subcritical or supercritical Hopf bifurcations, as sketched in Fig. $2[26]$.

2. Parametric excitation terms of the form $c \dot{\eta}_{2 n} \dot{\eta}_{n}$ or $c \eta_{2 n} \dot{\eta}_{n}$ are also known to be able to create a subcritical Hopf bifurcation and triggered limit cycles, as pictured in Fig. 2(a), depending 
on the sign of the coefficient $c$, i.e., $c<0$ for triggering, and $c>0$ for non-triggering [27].

Examining the nonlinear terms in the equation for the first acoustic mode, which is reproduced below from Eq. (2),

$$
\ddot{\eta}_{1}+\left[-2 \hat{\alpha}_{1}+\hat{C}_{11}^{(2)} \dot{\eta}_{2}\right] \dot{\eta}_{1}+\left[\left(1-2 \hat{\theta}_{1}\right)+\hat{D}_{11}^{(2)} \eta_{2}\right] \eta_{1}=[\text { other RHS terms }]
$$

it can be observed that there are no nonlinear damping terms, but the second order gasdynamic term $\hat{C}_{11}^{(2)} \dot{\eta}_{2} \dot{\eta}_{1}$ does indeed act as a parametric excitation of the desired form as listed in 2 . above. (The other parametric excitation term $\hat{D}_{11}^{(2)} \eta_{2} \eta_{1}$ is clearly not of the desired form.) However, on using the expressions following Eq. (1), the coefficient $\hat{C}_{11}^{(2)}$ can be evaluated to be

$$
\hat{C}_{11}^{(2)}=(3-2 \gamma) / 2 \gamma
$$

which is usually positive, where $\gamma$ is the ratio of specific heats. The term $\hat{C}_{11}^{(2)} \dot{\eta}_{2} \dot{\eta}_{1}$, thus, turns out to be a parametric excitation of the non-triggering type. It follows that second order gasdynamic nonlinearities, as they exist, are incapable of inducing triggered limit cycles. As an academic exercise, one may choose an arbitrary non-physical value of $\gamma$ that makes the coefficient $\hat{C}_{11}^{(2)}$ negative; then, triggered limit cycles can indeed be observed [29]. In summary, triggered limit cycles observed in solid propellant rockets cannot be explained by modeling the second order gasdynamic nonlinearities alone.

The most promising source for the triggering mechanism then appears to be nonlinear combustion. The approximate formulation of Eq. (1) already accounts for contributions from linear combustion processes. Nonlinear combustion phenomena can be included in the coupled oscillator system of Eq. (1) by introducing additional terms $F_{n}^{n c}$ representing pressure and velocity coupling effects. The modal equations for the set of coupled oscillators then appear as

$$
\begin{aligned}
\ddot{\eta}_{n}-2 \hat{\alpha}_{n} \dot{\eta}_{n}+n\left(n-2 \hat{\theta}_{n}\right) \eta_{n}= & -\sum_{i=1}^{n-1}\left(\hat{C}_{n i}^{(1)} \dot{\eta}_{i} \dot{\eta}_{n-i}+\hat{D}_{n i}^{(1)} \eta_{i} \eta_{n-i}\right) \\
& -\sum_{i=1}^{\infty}\left(\hat{C}_{n i}^{(2)} \dot{\eta}_{i} \dot{\eta}_{n+i}+\hat{D}_{n i}^{(2)} \eta_{i} \eta_{n+i}\right)+F_{n}^{n c}
\end{aligned}
$$

where $\eta_{n}$ is again the amplitude of the $n^{\text {th }}$ acoustic mode. Culick et al [19] and Burnley [22] considered the nonlinear pressure coupling terms in the Levine-Baum model as a possible candidate for the creation of triggered limit cycles. They observed that the nonlinear pressure coupling terms did indeed cause triggering, but the required values of the coefficients of these terms turned out to be unrealistically large. Their observations can now be explained by noting that the nonlinear pressure coupling terms in the Levine-Baum model are in fact second order parametric excitation terms with a negative coefficient (see Eq. (33) of Culick et al [19]), and, hence, of the type that can cause triggering. Then, for a first mode instability problem with second order gasdynamics and nonlinear pressure coupling, the parametric excitation terms in the equation for the first acoustic mode appear as $\left(\hat{C}_{11}^{(2)}+C_{1}^{p c}\right) \dot{\eta}_{2} \dot{\eta}_{1}$, where $C_{1}^{p c}$ is the coefficient of the pressure coupling term. Now, the combined coefficient $\left(\hat{C}_{11}^{(2)}+C_{1}^{p c}\right)$ is required to be negative for triggering to occur, and although $C_{1}^{p c}$ is known to be negative, it clearly needs to be large enough to overcome the positive value due to $\hat{C}_{11}^{(2)}$. Unfortunately, for reasonable values of $C_{1}^{p c}$, the combined coefficient is still positive, 
and, as a result, the nonlinear pressure coupling model does not lead to triggering. This leaves us to consider velocity coupling models as a possible candidate to explain the occurrence of triggered instability.

\section{Velocity Coupling Models}

Levine and Baum [17] suggested a velocity coupling function of the form $F_{n}^{n c}=f\left(\dot{\eta}_{n}\right) \dot{\eta}_{n}$, with $f\left(\dot{\eta}_{n}\right)=C_{n}^{v c}\left|\dot{\eta}_{n}\right|$, to model the nonlinear combustion response to an acoustic velocity parallel to the burning surface. The equation for the first acoustic mode, with second order gasdynamics and the Levine-Baum velocity coupling model, is then of the form

$$
\ddot{\eta}_{1}+\left[-2 \hat{\alpha}_{1}+\hat{C}_{11}^{(2)} \dot{\eta}_{2}+C_{1}^{v c}\left|\dot{\eta}_{1}\right|\right] \dot{\eta}_{1}+\left[\left(1-2 \hat{\theta}_{1}\right)+\hat{D}_{11}^{(2)} \eta_{2}\right] \eta_{1}=[\text { other RHS terms }]
$$

The velocity coupling function in Eq. (5) represents a nonlinear damping mechanism, and can, therefore, be expected to create triggered limit cycles at onset of first mode combustion instability. This is confirmed by computing equilibrium points and limit cycles for a four-mode truncation of the coupled oscillator system Eq. (4) for the data in Table 1. The velocity coupling function in the equation for the first acoustic mode is taken as shown in Eq. (5) with $C_{1}^{v c}=0.2$. Results for the first mode amplitude are plotted in Fig. 3 for varying values of the parameter $\hat{\alpha}_{1}$. Triggered limit cycles are clearly seen in Fig. 3 at a subcritical Hopf bifurcation, similar to that sketched in the schematic bifurcation diagram of Fig. 2(a). Thus, the Levine-Baum velocity coupling model along with the second order gasdynamic nonlinearities is adequate to capture triggered limit cycles in solid propellant combustion systems. However, from a qualitative point of view, the dynamics represented by the bifurcation diagram in Fig. 3 is not entirely satisfactory. This is because the stable triggered limit cycles terminate at some positive value of $\hat{\alpha}_{1}$ beyond which there are no stable equilibrium or limit cycle solutions. This implies that, where there are no stable solutions, the slightest perturbation will cause the modal amplitudes to eventually grow to infinity. Such dynamical behavior is clearly non-physical and must be eliminated.

Levine and Baum [17] also suggested that threshold effects that had been observed experimentally be incorporated in the velocity coupling function. Burnley and Culick [21] modified the LevineBaum velocity coupling function by introducing a dead zone to obtain an ad hoc velocity coupling function with a threshold, as follows:

$$
\begin{array}{ll}
f\left(\dot{\eta}_{1}\right)=0, & \left|\dot{\eta}_{1}\right|<\left|\dot{\eta}_{1}^{t}\right| \\
f\left(\dot{\eta}_{1}\right)=C_{1}^{v c}\left|\dot{\eta}_{1}-\dot{\eta}_{1}^{t}\right|, & \left|\dot{\eta}_{1}\right| \geq\left|\dot{\eta}_{1}^{t}\right|
\end{array}
$$

where $\dot{\eta}_{1}^{t}$ is the threshold value of $\dot{\eta}_{1}$. Computation of equilibrium and limit cycle solutions is carried out as before, but with the Burnley-Culick velocity coupling function in Eq. (6) instead of the Levine-Baum model. The parameter $C_{1}^{v c}$ in Eq. (6) is retained unchanged, i.e., $C_{1}^{v c}=0.2$, and a threshold value of $\dot{\eta}_{1}^{t}=0.02$ is chosen. A plot of the first mode amplitude for this case with varying values of the parameter $\hat{\alpha}_{1}$ is shown in Fig. 4, where triggered limit cycles of the form sketched in Fig. 2(b) may be observed at a supercritical Hopf bifurcation. However, it is known that functions $f\left(\dot{\eta}_{1}\right)$ that are approximately quadratic in shape, e.g., the Levine-Baum function, show triggered limit cycles of the subcritical type, while those that are approximately quartic (fourth-order), like the Burnley-Culick function, show triggered limit cycles of the supercritical type [26]. It is not 
difficult to come up with velocity coupling models with no threshold, but with an approximately quartic function $f\left(\dot{\eta}_{1}\right)$, that also produce triggered limit cycles of the supercritical type as in Fig. 4 [30]. Thus, the change from subcritical triggering in Fig. 3 to supercritical triggering in Fig. 4 cannot be attributed to the threshold effect in the Burnley-Culick velocity coupling function. Besides, the non-physical dynamical behavior seen over a range of positive values of $\hat{\alpha}_{1}$ in Fig. 3 persists in Fig. 4 as well.

To resolve this issue, we go back to the bifurcation diagram of Fig. 3 and the Levine-Baum velocity coupling model. It is clear that the qualitative dynamics of triggered limit cycles at onset of first mode combustion instability at the subcritical Hopf bifurcation is adequately captured in Fig. 3 . This means that the form of the Levine-Baum velocity coupling function is appropriate for small $\dot{\eta}_{1}$, i.e., near the region of onset of instability. The non-physical dynamics in Fig. 3 occurs for larger values of $\eta_{1}$ and $\dot{\eta}_{1}$, which implies that the form of the Levine-Baum velocity coupling model requires to be corrected for large $\dot{\eta}_{1}$, without affecting its shape in the neighborhood of $\dot{\eta}_{1}=0$. The lowest order correction term to the Levine-Baum function which meets these requirements is a quadratic term with value zero at $\dot{\eta}_{1}=0$, slope zero at $\dot{\eta}_{1}=0$, and a magnitude that subtracts from the value of the function for large $\dot{\eta}_{1}$. The new velocity coupling function, with such a quadratic term included, can be expressed as

$$
f\left(\dot{\eta}_{1}\right)=C_{1}^{v c}\left|\dot{\eta}_{1}\right|-D_{1}^{v c}\left|\dot{\eta}_{1}\right|^{2}
$$

For a particular choice of the coefficients, $C_{1}^{v c}=0.2$ and $D_{1}^{v c}=0.8$, the shape of the new velocity coupling function appears as plotted in Fig. 5. Surprisingly, the nonlinear function in Fig. 5 naturally shows a threshold character, but one that is quite different from the Burnley-Culick function. Computations are now carried out for equilibrium solutions and limit cycle amplitudes under identical conditions as was done for Fig. 3, but with the new velocity coupling function in Eq. (7) instead of the Levine-Baum model. Results for the first mode amplitude with varying parameter $\hat{\alpha}_{1}$ are shown in Fig. 6. As expected, the subcritical Hopf bifurcation in Fig. 6 is identical to that in Fig. 3, and the qualitative dynamics of the triggered limit cycles in the vicinity of the Hopf bifurcation point, i.e., for small $\eta_{1}$ and $\dot{\eta}_{1}$, remains unchanged. However, the stable limit cycles persist for all positive values of the parameter $\hat{\alpha}_{1}$, and the non-physical dynamical behavior in Fig. 3 is, therefore, eliminated in Fig. 6. Thus, the new velocity coupling function in Fig. 5 provides a satisfactory picture of the qualitative dynamics of the triggered limit cycles created at onset of combustion instability. In addition, the new velocity coupling function that is derived from dynamical considerations naturally satisfies the physical requirement of having a threshold character.

\section{Conclusions}

Several questions regarding the modeling and dynamics of acoustic waves in combustion chambers have been addressed in this paper using the approximate analysis originally developed by Culick. First among these is the question of modal truncation, i.e., how many modes need to be retained in a truncated model of the coupled oscillator equations in order to predict the qualitative dynamics of the acoustic waves correctly. Previous studies of first and second mode instabilities arbitrarily chose to retain between two and sixteen modes. We have now shown that a first mode instability requires a minimum of four modes in the modal truncation, while for a second mode instability, one 
needs to retain at least the first eight modes. Secondly, it has been widely believed from previous studies that the approximate analysis with only second order gasdynamic nonlinearities could not show triggered limit cycles. This has now been theoretically established by recognizing that second order gasdynamics does not contribute either nonlinear damping or parametric excitation terms in the form required to cause triggered limit cycles. Finally, nonlinear combustion mechanisms for triggering based on pressure and velocity coupling models have been studied. Results from a previous study which suggested that pressure coupling does not lead to triggering have now been explained. Velocity coupling models have been shown to induce triggered instability due to a nonlinear damping mechanism. Velocity coupling models used in the past have been examined, and a new velocity coupling function has been derived that captures the qualitative dynamics at onset of triggered instability. Interestingly, our velocity coupling function naturally shows a threshold nature unlike previous velocity coupling models that had an artificially imposed threshold character.

\section{Acknowledgments}

This work was supported in part by the California Institute of Technology; partly by the Caltech Multidisciplinary University Research Initiative under Grant No. N00014-95-1-1338 (Dr. Judah Goldwasser, Program Manager); partly by the Department of Energy Advanced Gas Turbine Systems Research (AGTSR) Program under Subcontract No. 98-02-SR072 (Dr. Larry Golan, Program Manager); and partly by the Air Force Office of Scientific Research (AFOSR) under Grant No. F49620-99-1-0118 (Dr. Mitat Birkan, Program Manager).

\section{References}

[1] Chu, B.-T., and Kovasznay, L.S.G., "Nonlinear Interactions in a Viscous Heat-Conducting Compressible Gas," Journal of Fluid Mechanics, Vol. 3, No. 5, 1957, pp. 494-512.

[2] Baum, J.D., Levine, J.N., and Lovine, R.L., "Pulse Triggered Nonlinear Combustion Instability in Solid Rocket Motors," AIAA Journal, Vol. 22, No. 10, 1984, pp. 1413-1419.

[3] Baum, J.D., Levine, J.N., and Lovine, R.L., "Pulsed Instability in Rocket Motors: A Comparision Between Predictions and Experiments," Journal of Propulsion and Power, Vol. 4, No. 4, 1988, pp. 308-316.

[4] Lieuwen, T.C,, "Experimental Investigation of Limit Cycle Oscillations in an Unstable Gas Turbine Combustor," Journal of Propulsion and Power, Vol. 18, No. 1, 2002, pp. 61-67.

[5] Flandro, G.A., "Effects of Vorticity on Rocket Combustion Stability," Journal of Propulsion and Power, Vol. 11, No. 4, 1995, pp. 607-625.

[6] Garcia-Schafer, J.E., and Linan, A., "Longitudinal Acoustic Instabilities in Slender Solid Propellant Rockets: Linear Analysis," Journal of Fluid Mechanics, Vol. 437, 2001, pp. 229-254.

[7] Culick, F.E.C., "Some Recent Results for Nonlinear Acoustics in Combustion Chambers," AIAA Journal, Vol. 32, No. 1, 1994, pp. 146-169. 
[8] Culick, F.E.C., "Combustion Instabilities in Propulsion Systems," Unsteady Combustion, F.E.C. Culick et al (eds.), Kluwer Academic Publishers, Netherlands, 1996, pp. 173-241.

[9] Culick, F.E.C., "Nonlinear Growth and Limiting Amplitude of Acoustic Oscillators in Combustion Chambers," Combustion Science and Technology, Vol. 3, No. 1, 1971, pp. 1-16.

[10] Culick, F.E.C., "Nonlinear Behavior of Acoustic Waves in Combustion Chambers, Parts I and II," Acta Astronautica, Vol. 3, 1976, pp. 714-757.

[11] Culick, F.E.C., and Yang, V., "Prediction of the Stability of Unsteady Motions in Solid Propellant Rocket Motors," Nonsteady Burning and Combustion Stability of Solid Propellants, L. de Luca, E.W. Price, and M. Summerfield (eds.), AIAA, Washington, 1992.

[12] Awad, E., and Culick, F.E.C., "On the Existence and Stability of Limit Cycles for Longitudinal Acoustic Modes in a Combustion Chamber," Combustion Science and Technology, Vol. 46, No. 6, 1986, pp. 195-222.

[13] Paparizos, L.G., and Culick, F.E.C., "The Two-Mode Approximation to Nonlinear Acoustics in Combustion Chambers, I. Exact Solution for Second Order Acoustics," Combustion Science and Technology, Vol 65, 1989, pp. 39-65.

[14] Yang, V., Kim, S.I., and Culick, F.E.C., "Triggering of Longitudinal Pressure Oscillations in Combustion Chambers, I: Nonlinear Gasdynamics," Combustion Science and Technology, Vol. 72 , No. 5, 1990, pp. 183-214.

[15] Zinn, B.T., "A Theoretical Study of Nonlinear Combustion Instability in Liquid Propellant Rocket Engines," AIAA Journal, Vol. 6, No. 10, 1968, pp. 1966-1972.

[16] Culick, F.E.C., "Stability of Longitudinal Oscillations with Pressure and Velocity Coupling in a Solid Propellant Rocket," Combustion Science and Technology, Vol. 2, No. 4, 1970, pp. 179201.

[17] Levine, J.N., and Baum, J.D., "A Numerical Study of Nonlinear Instability Phenomena in Solid Rocket Motors," AIAA Journal, Vol. 21, No. 4, 1983, pp. 557-564.

[18] Jahnke, C.C., and Culick, F.E.C., "Application of Dynamical Systems Theory to Nonlinear Combustion Instabilities," Journal of Propulsion and Power, Vol. 10, No. 4, 1994, pp. 508-517.

[19] Culick, F.E.C., Burnley, V., and Swenson, G., "Pulsed Instabilities in Solid Propellant Rockets," Journal of Propulsion and Power, Vol. 11, No. 4, 1995, pp. 657-665.

[20] Ma, Y., van Moorhem, W.K., and Shorthill, R.W., "Experimental Investigation of Velocity Coupling in Combustion Instability," Journal of Propulsion and Power, Vol. 7, No. 5, 1991, pp. 692-699.

[21] Burnley, V.S., and Culick, F.E.C., "Influence of Random Excitations on Acoustic Instabilities in Combustion Chambers," AIAA Journal, Vol. 38, No. 8, 2000, pp. 1403-1410.

[22] Burnley, V.S., "Nonlinear Combustion Instabilities and Stochastic Sources," Ph. D. Thesis, California Inst. of Technology, Pasadena, CA, 1996. 
[23] Poinsot, T., Bourienne, F., Candel, S.H., and Esposito, E., "Suppression of Combustion Instabilities by Active Control," Journal of Propulsion and Power, Vol. 5, No. 1, 1989, pp. 14-20.

[24] McManus, K.R., Poinsot, T., and Candel, S.M., "A Review of Active Control of Combustion Instabilities," Progress in Energy and Combustion Science, Vol. 19, 1993, pp. 1-29.

[25] Ananthkrishnan, N., and Sudhakar, K., "Characterization of Periodic Motions in Aircraft Lateral Dynamics," Journal of Guidance, Control, and Dynamics, Vol. 19, No. 3, 1996, pp. 680685.

[26] Ananthkrishnan, N., Sudhakar, K., Sudershan, S., and Agarwal, A., "Application of Secondary Bifurcations to Large Amplitude Limit Cycles in Mechanical Systems," Journal of Sound and Vibration, Vol. 215, No. 1, 1998, pp. 183-188.

[27] Ananthkrishnan, N., Sudershan, S., Sudhakar, K., and Verma, A., "Large Amplitude Limit Cycles in Resonantly Coupled Oscillators," Journal of Sound and Vibration, Vol. 231, No. 5, 2000, pp. 1377-1382.

[28] Doedel, E.J., Wang, X.J., Fairgrieve, T.F., Champneys, A.R., Kuznetsov, Y.A., and Sandstede, B., "AUTO97: Continuation and Bifurcation Software for Ordinary Differential Equations (with HomCont)," California Inst. of Technology, Pasadena, CA, 1998.

[29] Ananthkrishnan, N., "Qualitative Dynamics of Nonlinear Acoustic Waves in a Combustion Chamber, I: Modal Truncation and Triggering," Guggenheim Jet Propulsion Center Documents on Active Control of Combustion Instabilities, CI 00-03, California Inst. of Technology, Pasadena, CA, 2000.

[30] Ananthkrishnan, N., "Qualitative Dynamics of Nonlinear Acoustic Waves in a Combustion Chamber, III: Velocity Coupling Models," Guggenheim Jet Propulsion Center Documents on Active Control of Combustion Instabilities, CI 01-01, California Inst. of Technology, Pasadena, CA, 2001. 
Table 1. Data for parameters $\alpha_{n}$ and $\theta_{n}$.

\begin{tabular}{lrrrrrr}
\hline \hline Mode & 1 & 2 & 3 & 4 & 5 & 6 \\
\hline$\alpha_{n}, 1 / \mathrm{s}$ & Free & -324.8 & -583.6 & -889.4 & -1262.7 & -1500.0 \\
$\theta_{n}, \mathrm{rad} / \mathrm{s}$ & 12.9 & 46.8 & -29.3 & -131.0 & -280.0 & -300.0 \\
\hline \hline
\end{tabular}

Table 2. Inter-modal energy transfers.

\begin{tabular}{cll}
\hline \hline Mode number & Energy transfer up the modes & Reverse energy transfer \\
\hline 1 & & $\mathbf{1 2}, 23,34,45,56,67,78$ \\
2 & 11 & $13, \mathbf{2 4}, 35,46,57,68$ \\
3 & 12,21 & $14,25, \mathbf{3 6}, 47,58$ \\
4 & $13,22,31$ & $15,26,37, \mathbf{4 8}$ \\
5 & $14,23,32,41$ & $16,27,38$ \\
6 & $15,24,33,42,51$ & 17,28 \\
7 & $16,25,34,43,52,61$ & 18 \\
8 & $17,26,35,44,53,62,71$ & \\
\hline \hline
\end{tabular}




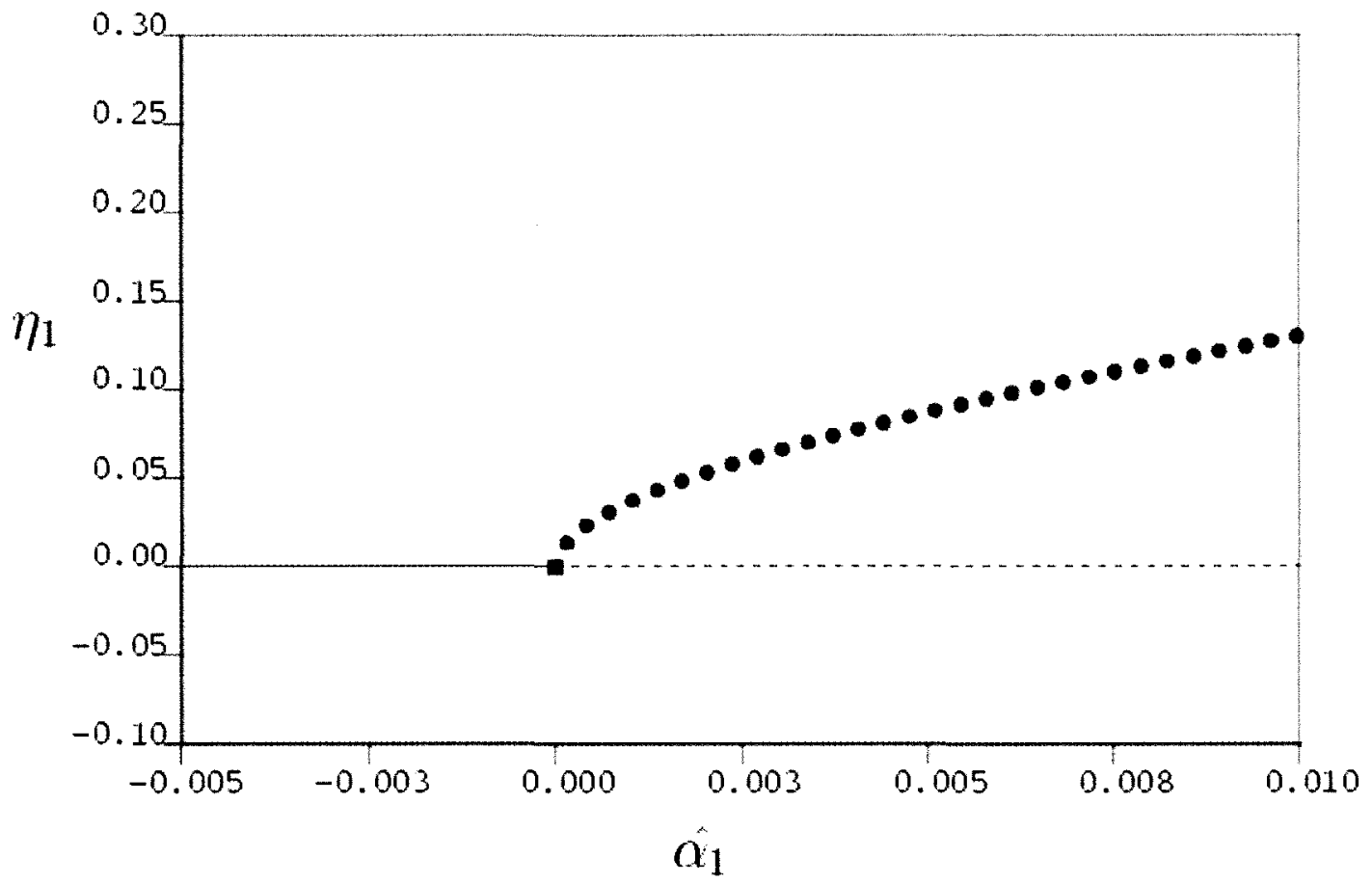

Fig. 1. Stable limit cycles at a supercritical Hopf bifurcation for a first mode instability with second order gasdynamics alone (full line - stable equilibrium, dashed line -- unstable equilibrium, filled circle - stable limit cycle, filled square - Hopf bifurcation). 


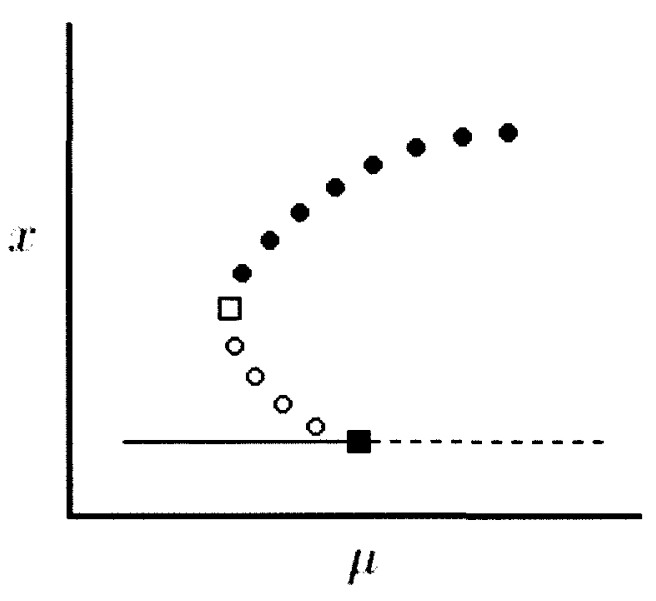

(a)

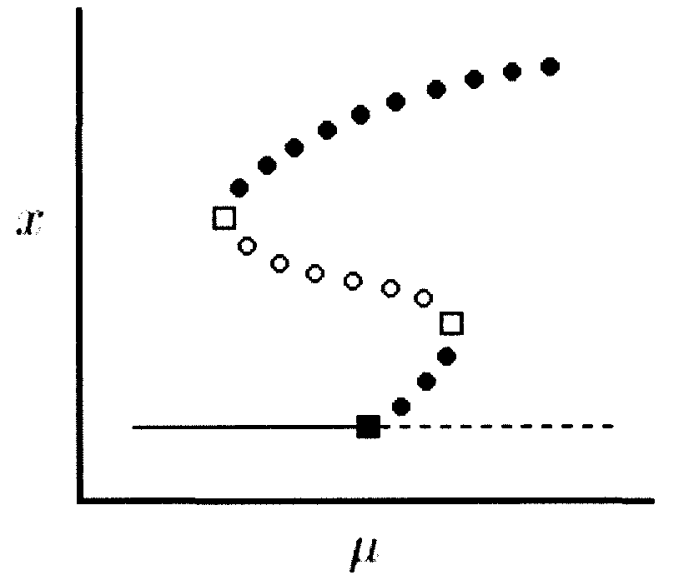

(b)

Fig. 2. Triggered limit cycles at (a) subcritical Hopf bifurcation, and (b) supercritical Hopf bifurcation (full line - stable equilibrium, dashed line - unstable equilibrium, filled circle stable limit cycle, empty circle - unstable limit cycle, filled square - Hopf bifurcation, empty square - fold bifurcation).

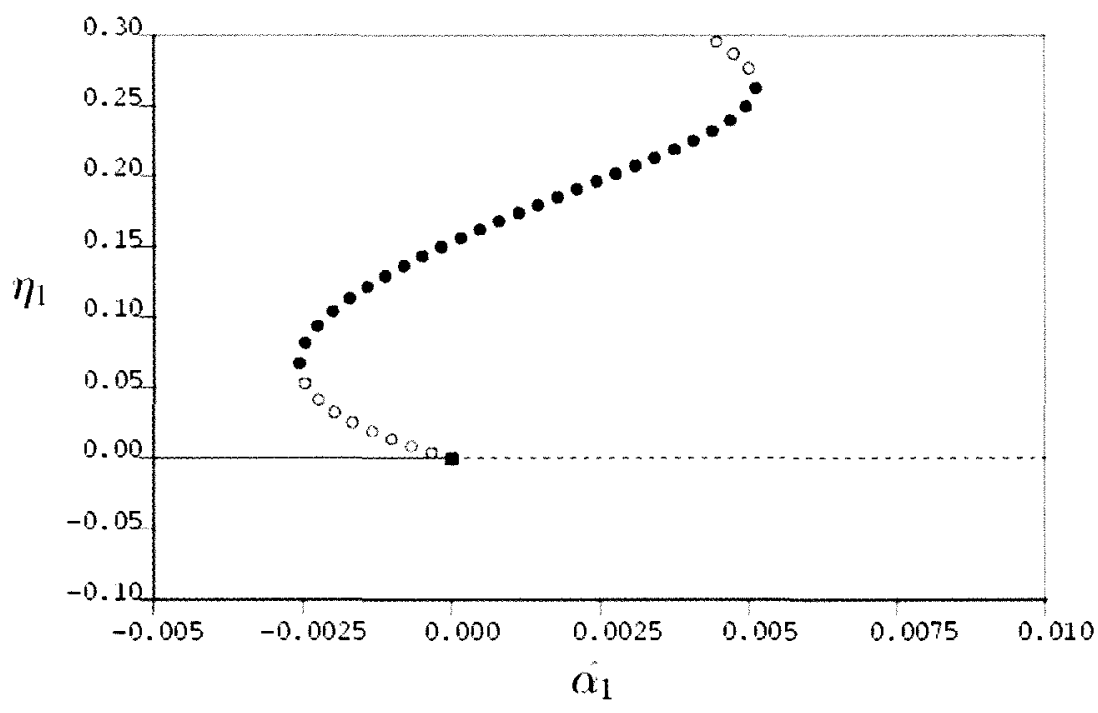

Fig. 3. Triggered limit cycles at a subcritical Hopf bifurcation for a first mode instability with second order gasdynamics and the Levine-Baum velocity coupling function (full line - stable equilibrium, dashed line - unstable equilibrium, filled circle - stable limit cycle, empty circle unstable limit cycle, filled square - Hopf bifurcation). 


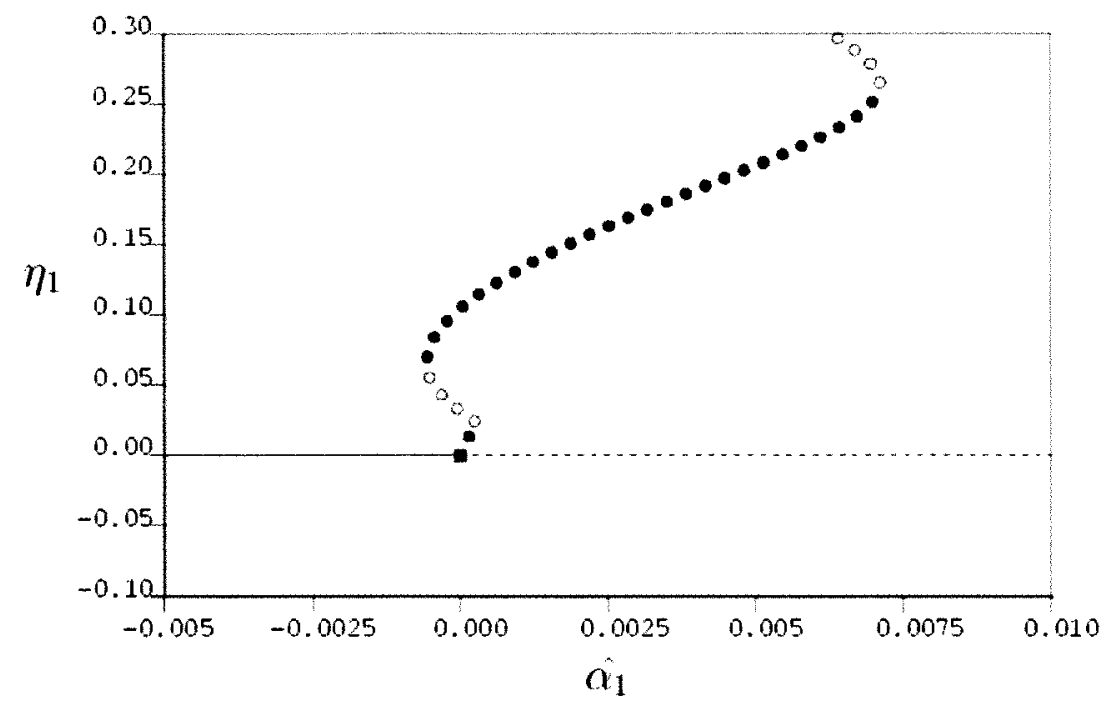

Fig. 4. Triggered limit cycles at a supercritical Hopf bifurcation for a first mode instability with second order gasdynamics and the Burnley-Culick threshold velocity coupling function (full line - stable equilibrium, dashed line - unstable equilibrium, filled circle - stable limit cycle, empty circle - unstable limit cycle, filled square - Hopf bifurcation).

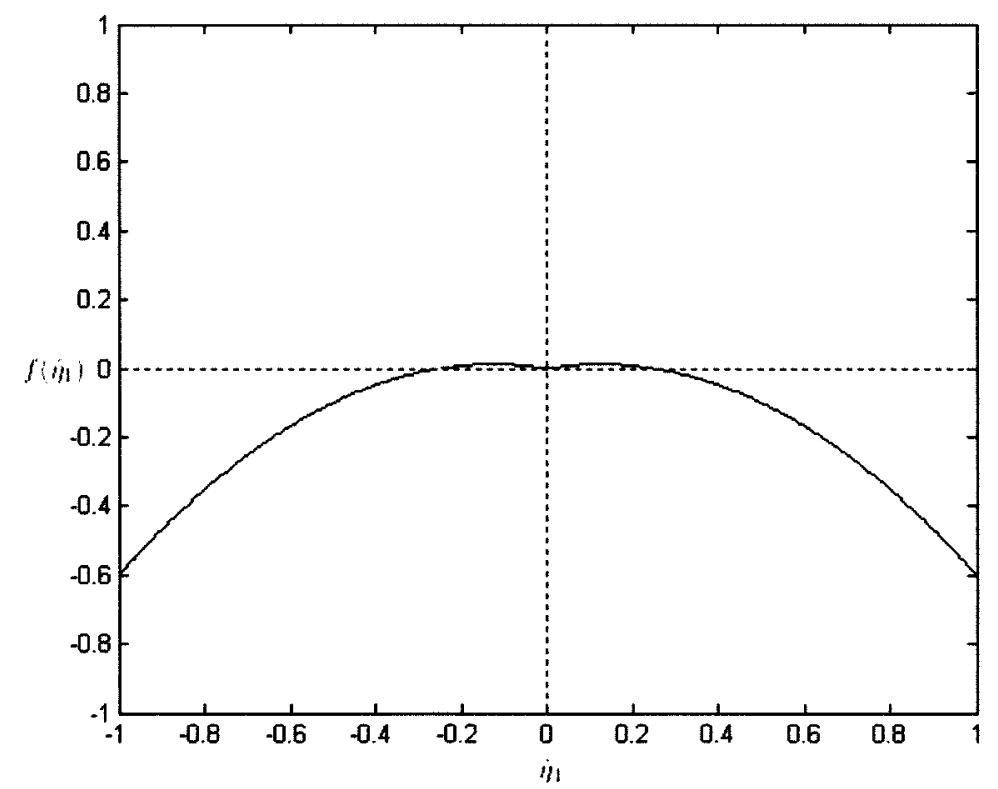

Fig. 5. The form of the velocity coupling function obtained by correcting the Levine-Baum function with a suitable quadratic term. 


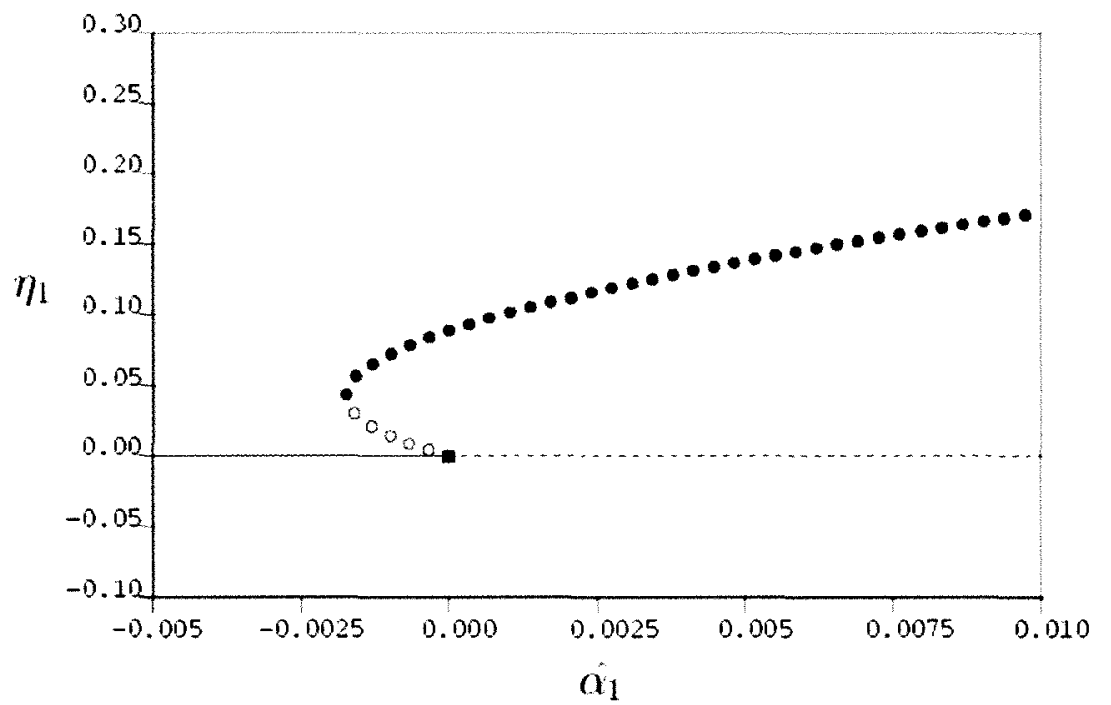

Fig. 6. Triggered limit cycles at a subcritical Hopf bifurcation for a first mode instability with second order gasdynamics and the threshold velocity coupling function in Fig. 5 (full line stable equilibrium, dashed line - unstable equilibrium, filled circle - stable limit cycle, empty circle - unstable limit cycle, filled square - Hopf bifurcation). 